\title{
Associação do Alótipo Raro C4A6 do Sistema Complemento com a Doença Cardíaca Reumática ${ }^{*}$
}

\section{Association of the C4A6 Rare Allotype with Rheumatic Heart Disease}

\author{
Iara Jose de Messias-Reason ${ }^{(1)}$, Ednéia Cavalcanti ${ }^{(1)}$ e Sebastião Cézar Radominski ${ }^{(2)}$
}

\section{RESUMO}

Objetivo: determinar se os alótipos do Fator B (BF), C2 e C4 (C4A e C4B) do sistema complemento poderiam ser marcadores para a doença cardíaca reumática (DCR) na população brasileira. Métodos: foram estudados 49 pacientes com DCR crônica. Os controles incluíram 65 indivíduos saudáveis, não aparentados, pareados com a amostra dos pacientes de acordo com o sexo, idade e grupo racial. Os alótipos de BF, C2, C4A e C4B foram determinados por técnicas-padrão, incluindo Western blot para a determinação das variantes de $\mathrm{C} 2$ e $\mathrm{C} 4$, utilizando-se anticorpos monoclonais e policlonais. Resultados: este estudo demonstrou um significante aumento do alelo raro $\mathrm{C}_{4} \mathrm{~A}^{*} 6(\mathrm{p}=0,003 \mathrm{RR}=11,85)$ e diminuição de $\mathrm{C}_{4} \mathrm{~A}^{*} 3$ nos pacientes, quando comparados com os controles. Além disso, alelos nulos de $\mathrm{C} 4$ e raros de $\mathrm{C} 4$ e $\mathrm{BF}$ foram observados com maior freqüência nos pacientes. Conclusões: considerando-se que foram avaliados somente pacientes com DCR, mais estudos serão necessários para que se possa esclarecer se o alelo C4A6 pode ser um marcador da forma cardíaca ou da própria doença.

Palavras-chave: complemento, variabilidade alotípica, doença cardíaca reumática, febre reumática.

\section{INTRODUÇÃO}

Embora a prevalência e a gravidade da febre reumática (FR) tenham diminuído consideravelmente nas últimas décadas na América do Norte e Europa, esta doença continua a ocorrer em proporção epidêmica em muitos países em desenvolvimento, incluindo o Brasil. Aproximadamente $30 \%$ dos pacientes com FR desenvolvem a forma cardíaca

\begin{abstract}
Objective: to determine whether the allotypes of complement proteins Factor $B(B F), C 2, C 4 A$ and $C 4 B$ could be markers of rheumatic heart disease (RHD) in the Brazilian population. Methods: forty-nine patients with chronic RHD were studied. The controls included 65 healthy individuals, matched with the patients according to sex, age and ethnical background. $B F, C 2, C 4 A$ and $C 4 B$ allotypes were studied by standard techniques including Western blots for C2 and C4 variants with monoclonal and polyclonal antibodies. Results: this study showed a significantly elevated frequency of the rare C4A6 allotype $(p=0.03 R R=11.85)$ and a decrease of $C 4 A 3$ in the patients when compared to controls. In addition, $C 4$ null and $B F$ and C4 rare allotypes were more frequent in patients than in controls. Conclusions: considering that only RHD patients were included, further investigations are necessary in order to clarify whether C4A6 may be a marker of the cardiac form or of the disease itself.
\end{abstract}

Keywords: complement, allotypes, rheumatic heart disease, rheumatic fever.

(DCR), que constitui a mais importante e séria manifestação da doença. No Brasil, de acordo com dados oficiais do governo, em 1990 aproximadamente 40\% das cirurgias cardíacas foram realizadas para corrigir seqüelas cardíacas causadas pela DCR, representando um grave problema de saúde neste país.

Embora haja evidências de que uma potencial causa da FR e da DCR seja uma resposta imune anormal a uma

\footnotetext{
* Disciplina de Reumatologia, Departamento de Clínica Médica, Hospital de Clínicas da Universidade Federal do Paraná, Curitiba, Brasil. Este trabalho contou com o apoio do Fundo de Auxílio à Pesquisa e Ensino em Reumatologia da Sociedade Brasileira de Reumatologia. Recebido em 27/1 1/2003. Aprovado, após revisão, em 28/4/2004.

1. Laboratório de Imunopatologia, Departamento de Patologia Médica, Hospital de Clínicas da Universidade Federal do Paraná, Curitiba, Brasil.

2. Disciplina de Reumatologia, Departamento de Clínica Médica, Hospital de Clínicas da Universidade Federal do Paraná, Curitiba, Brasil.

Endereço para correspondência: Iara de Messias Reason. Departamento de Patologia Médica/UFPR. Rua Padre Camargo, 280, CEP 80.069-900, Curitiba-PR, Brasil. E-mail: iaramessias@yahoo.com.br
} 
infecção estreptocócica, os mecanismos precisos envolvidos na sua patogênese ainda não são bem conhecidos ${ }^{(1)}$. Vários estudos apontam a influência genética na susceptibilidade da FR e da DCR ${ }^{(1-3)}$. Além da influência familiar ${ }^{(2-4)}$, associações significantes têm sido encontradas em diferentes populações, especialmente com antígenos da classe II do complexo principal de histocompatibilidade $(\mathrm{MHC})^{(5-7)}$. Vários estudos demonstram significante associação entre diferentes alótipos do complemento e doenças autoimunes e infecciosas, tais como artrite reumatóide, esclerose múltipla, lúpus eritematoso sistêmico, doença de Chagas e hanseníase ${ }^{(8-12)}$. Os componentes do complemento $\mathrm{C} 2$, fator B (BF) e C4 estão entre as proteínas codificadas pela região da classe III do MHC. O C4 consiste em duas isoproteínas designadas como C4A e C4B, codificadas por dois loci interligados; $\mathrm{BF}$ e C2 são codificados por apenas um gene ${ }^{(13)}$. A proteína BF é essencial na ativação da via alternativa do complemento, enquanto que C2 e C4 constituem a principal enzima ativadora de $\mathrm{C} 3$ da via clássica. Conseqüentemente, estes componentes desempenham um importante papel na ativação do complemento e na geração das subseqüentes atividades biológicas mediadas por este sistema, tanto na resposta imunológica quanto nas reações inflamatórias. Neste estudo investigou-se se os alótipos de BF, C2 e C4 poderiam ser marcadores para a DCR na população brasileira.

\section{PACIENTES E MÉTODOS}

Foram estudados 49 pacientes atendidos pela clínica de Reumatologia e Cardiologia do Hospital de Clínicas da Universidade Federal do Paraná (UFPR), com doença valvar cardíaca reumática, apresentando história prévia de FR. O envolvimento cardíaco foi baseado em achados clínicos e laboratoriais, comprovados através de radiografia do tórax, eletrocardiografia, ecocardiografia, e, em alguns casos, cateterismo. Entre os 49 pacientes, 23 eram homens e 26 mulheres, com idade entre 13 e 50 anos (média de 24 anos). Desse total, 43 (88\%) eram de origem caucasóide, $5(10 \%)$ eram mulatos e $1(2 \%)$ era negro. Como controles, foram analisados 65 indivíduos saudáveis não aparentados (28 mulheres e 37 homens), com idade entre 18 e 54 anos (média de 28,8 anos). O grupo controle foi pareado com os pacientes de acordo com a origem étnica, sendo 57 (88\%) deles caucasóides, 6 (9\%) mulatos e $2(3 \%)$ negros. Este trabalho foi aprovado pelo Comitê de Ética de Pesquisa em Seres Humanos do Hospital de Clínicas da UFPR.
Soro - Foram coletados $10 \mathrm{ml}$ de sangue venoso de cada indivíduo, após consentimento informado. Depois de coaguladas, as amostras foram centrifugadas a $4^{\circ} \mathrm{C}$ e o soro dividido em alíquotas e armazenado a $-70^{\circ} \mathrm{C}$, até serem utilizadas.

Alotipagem do complemento - A tipagem de BF foi realizada através de eletroforese de alta voltagem em gel de agarose (HVAGE) e imunofixação com anticorpo antiBF humano (Atlantic Antibodies, Scarborough, ME, USA), de acordo com Geserick et al. ${ }^{(14)}$. A tipagem de C2 foi executada utilizando-se focalização isoelétrica, seguida por teste hemolítico funcional de acordo com Alper ${ }^{(15)}$ e por Western Blot como descrito por Uring Lambert et al. ${ }^{(16)}$. A tipagem de C4A e C4B foi determinada em plasma com EDTA, tratado com neuraminidase e carboxipeptidase B (Sigma, St Louis, MO, USA). Após eletroforese de alta voltagem (HVAGE), os alótipos de C4A e C4B foram determinados por imunofixação com anti-C4 humano (Atlantic Antibodies) e teste hemolítico ${ }^{(17)}$. Para a definição de alguns alelos, foram empregados eletroforese prolongada em gel e Western blot, usando anticorpos monoclonais específicos contra alelos de $\mathrm{C} 4 \mathrm{~A}$ e $\mathrm{C} 4 \mathrm{~B}^{(17,18)}$. A estimativa de alelos não expressos de C4A e C4B foi feita por avaliação semiquantitativa das placas de eletroforese e pelo método MASC (qui-quadrado mínimo) $)^{(19,20)}$.

\section{RESULTADOS}

A distribuição dos alótipos de $\mathrm{BF}$ e de $\mathrm{C} 2$ entre os pacientes de DCR e o grupo controle não apresentou nenhuma diferença estatisticamente significante (Figuras 1 e 2). $\mathrm{O}$ alótipo S07 foi observado em dois pacientes $(4,4 \%)$ no fenótipo SS07, e em um indivíduo controle $(1,5 \%)$ no fenótipo FS07. O outro alótipo incomum BFF1 foi observado em um indivíduo controle no fenótipo F1S. Além dos alótipos comuns $\mathrm{C} 2 \mathrm{C}$ e C2B, nenhum outro alótipo de $\mathrm{C} 2$ foi observado nos pacientes e controles.

Com relação à distribuição dos alótipos de C4A e C4B, foram observadas duas duplicações no locus $\mathrm{C} 4 \mathrm{~A}$, uma em um paciente e outra em um controle: C4A 3,12,1 e C4A 4,3,2 respectivamente (Figuras 3 e 4). Os alótipos raros C4B28 e B6 estavam presentes em dois diferentes pacientes, enquanto o B22 estava presente em um indivíduo controle. No locus C4A foi observada a diminuição de C4 A3 nos pacientes $(p=0,03 R R=0,3)$, entretanto este número não foi significante após correção para o número de alelos observados. Por outro lado, o alótipo raro C4 A6 esteve 
significativamente aumentado nos pacientes $(6 / 44 ; 13,6 \%)$ quando comparados aos controles $(0 / 65 ; \mathrm{p}=0,003$ $\mathrm{RR}=11,85)$. Além disso, o alótipo raro C4A5 esteve presente em um indivíduo controle. As freqüências de alelos de $\mathrm{C} 4$ não expressos $\left(\mathrm{A}^{*} \mathrm{Q} 0\right.$ e $\left.\mathrm{B}^{*} \mathrm{Q} 0\right)$ foram mais elevadas entre os pacientes $(\mathrm{AQ} 0=14 / 44 ; 31,8 \%$ e $\mathrm{BQ} 0=13 / 44$;

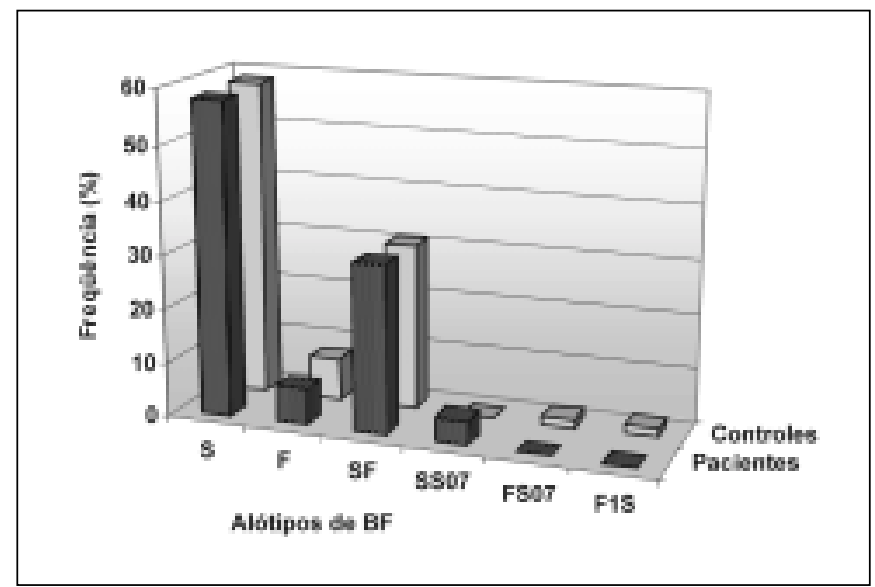

Figura 1 - Freqüência dos alótipos de BF nos pacientes com DCR e nos controles normais. Todos os alótipos de BF não mostraram diferença estatisticamente significante $(p>0,05)$.
$29,5 \%$ ) quando comparadas com as dos controles ( $\mathrm{AQ} 0=14 /$ $65 ; 2,01 \%$ e $\mathrm{BQ} 0=13 / 65 ; 20,0 \%)$, entretanto estes valores não foram estatisticamente significantes. Deficiência homozigótica para o locus C4A foi observada em um paciente e em um controle, enquanto que para o locus C4B foi observada em um paciente.

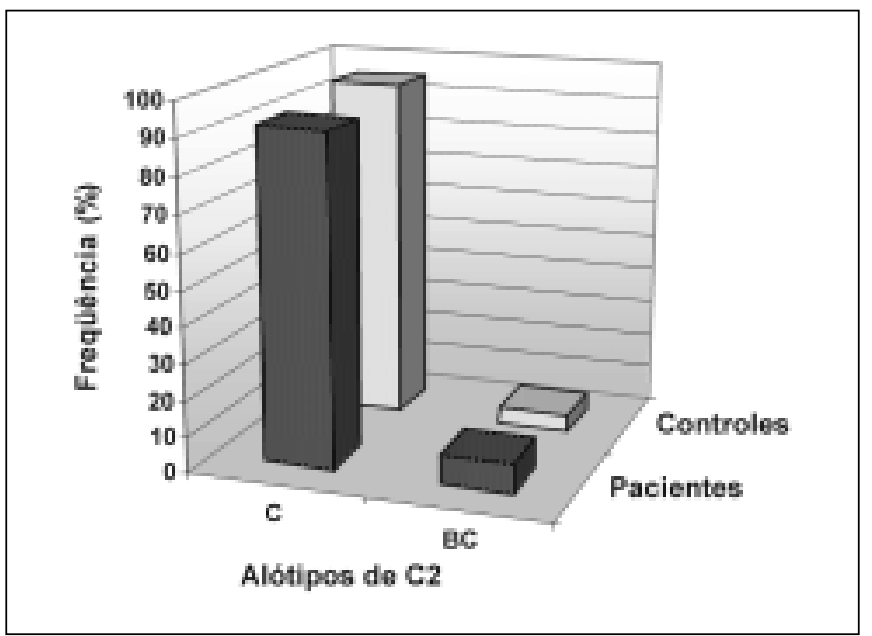

Figura 2 - Freqüência dos alótipos de C2 nos pacientes com DCR e nos controles normais. Não houve diferença estatisticamente significante na freqüência dos alótipos de C2 entre pacientes e controles $(p>0,05)$.

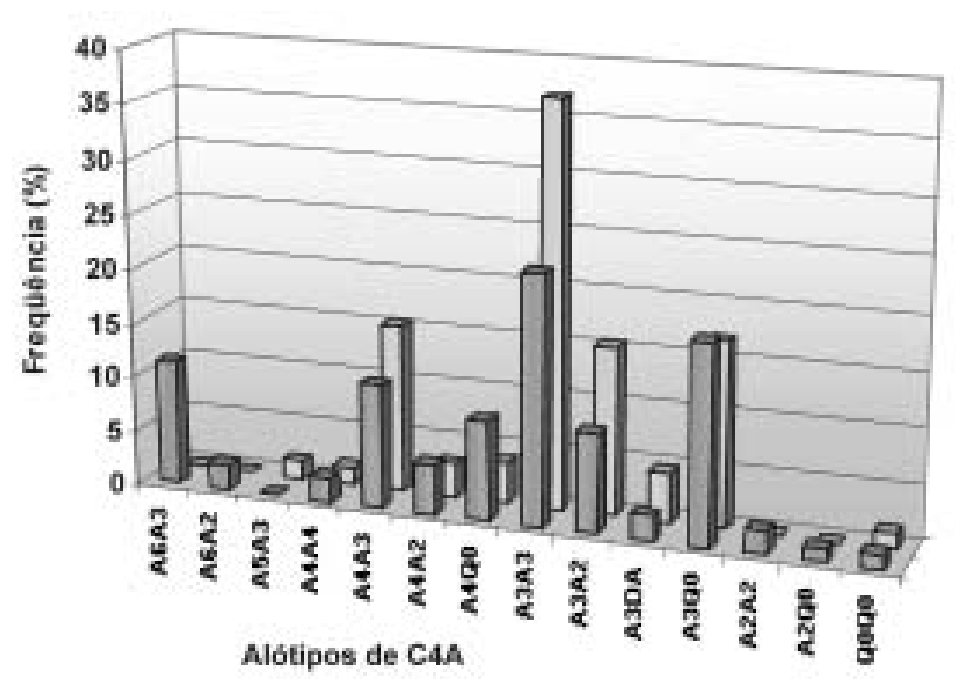

\section{口Pacientes \\ $\square$ Controles}

FIgURA 3 - Freqüência dos alótipos de C4A nos pacientes com DCR e nos controles normais. DA=alelo duplicado; Q0=alelo nulo; * p=0,003; RR=11.85. 


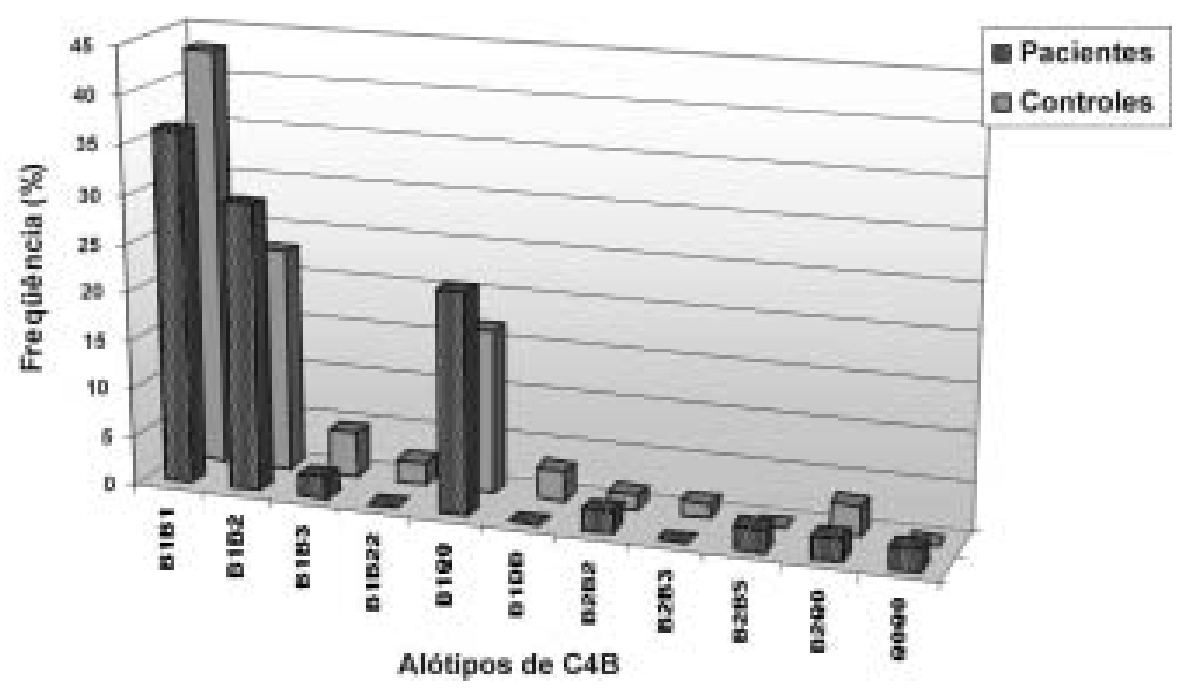

FIgURA 4 - Freqüência dos alótipos de C4B nos pacientes com DCR e nos controles normais. DB=alelo duplicado; Q0=alelo nulo; $p=n s$ para todos os alelos de C4B.

\section{DISCUSSÃO}

Acredita-se que a FR seja desencadeada por uma resposta imunológica anormal, contra antígenos do estreptococo do grupo $\mathrm{A}$, em indivíduos geneticamente suscetíveis. $\mathrm{O}$ fato de somente um pequeno número de indivíduos expostos a estes microorganismos desenvolverem a FR ou a DCR aponta para a existência de mecanismos naturais de resistência à doença em indivíduos com resposta imunológica eficiente ${ }^{(1,3)}$. Paralelamente, a diversidade nas manifestações clínicas sugere o envolvimento de vários fatores na susceptibilidade da doença, tais como fatores genéticos, sociais e econômicos, além daqueles relacionados com a virulência do estreptococo.

Após achados de estudos epidemiológicos e imunológicos, sugerindo que a doença se manifeste em pessoas predispostas, vários investigadores têm procurado estabelecer uma associação entre distintos marcadores genéticos e a FR/DCR ${ }^{(21-23)}$. Um dos achados mais interessantes foi o aumento significante do antígeno D8/17 dos linfócitos B em pacientes com FR, o qual se mostrou fortemente associado à doença em diferentes populações ${ }^{(21-24)}$.

Durante os últimos 30 anos, vários estudos têm analisado a associação dos antígenos do MHC com a FR. Estudos iniciais investigaram diferentes antígenos da classe $\mathrm{I}^{(25-27)}$. Entretanto, associações significantes foram demonstradas com os antígenos da Classe II em grupos raciais distintos ${ }^{(5,6)}$.
Os significantes achados relacionados com os antígenos DR têm sugerido que outros genes de susceptibilidade, situados próximos desta região do MHC, poderiam estar envolvidos na resposta imune anormal contra os antígenos estreptocócicos. Em razão da proximidade dos genes da classe III do complemento a esta região do MHC e a significante associação de diferentes alótipos do complemento com doenças auto-imunes descrita na literatura, a variabilidade alotípica destes componentes foi determinada num grupo de pacientes com DCR e controles.

No presente estudo, foi observada uma associação estatisticamente significante do alelo raro $\mathrm{C}_{4} \mathrm{~A}^{*} 6 \mathrm{com}$ a DCR. Além disso, a presença de alelos nulos de C4 e outros alelos raros de $\mathrm{BF}$ e $\mathrm{C} 4$ foi mais freqüentemente observada nos pacientes do que no grupo controle. Embora a presença de $\mathrm{C}_{4} \mathrm{~A}^{*} 6$ não tenha ocorrido na maioria dos pacientes investigados, sua freqüência foi significantemente maior quando comparada ao grupo controle $(\mathrm{p}=0,003 \mathrm{R} R=11,85)$ e com a população sul brasileira ${ }^{(28)}(13,63 \%$ vs 3,48\%). É possível que o alótipo $\mathrm{C}_{4} \mathrm{~A}^{*} 6$ seja relevante para um subgrupo de pacientes com DCR. Além disso, a maior freqüência de outros alótipos raros de C4 e de BF nos pacientes pode sugerir um papel imunogenético dos alótipos raros do complemento na DCR.

O sistema complemento é um dos primeiros mecanismos de defesa do hospedeiro e ocupa um importante papel tanto 
na opsonização quanto na lise de membranas celulares de patógenos, além de atuar como um sistema efetor de anticorpos. Recentemente, Saeland et al. ${ }^{(29)}$ demonstraram que o complemento tem um papel central na proteção mediada por anticorpos contra a infecção pelo Streptococcus pneumoniae "in vivo". Estreptococos reumatogênicos apresentam mecanismos de evasão à atividade lítica do complemento, através da aquisição de moléculas regulatórias do hospedeiro como fator $\mathrm{H}$ e proteína ligante de $\mathrm{C} 4 \mathrm{~b}^{(30)}$. Além disso, diferentes cepas secretam proteínas/enzimas que, além de inibir a ativação do complemento, inibem a quimiotaxia e ativação fagocitária induzida por produtos de ativação do complemento, como C5a e C3a ${ }^{(30)}$. As proteínas $\mathrm{BF}, \mathrm{C} 2 \mathrm{e} \mathrm{C} 4$ ocupam um papel essencial nas primeiras etapas da ativação do complemento e na subseqüente opsonização, transporte e solubilização de complexos imunes ${ }^{(8)}$. Sabe-se que diferentes alótipos de BF, C2 e C4 apresentam diferenças em suas atividades funcionais ou em seus níveis plasmáticos.

A associação de doenças com alelos silenciosos e raros dessas proteínas sugere possível diferença funcional entre os alótipos na ação do complemento contra agentes infecciosos e nas reações imunes ${ }^{(10,31)}$. Um aumento do alelo

\section{REFERÊNCIAS}

1. Senitzer D, Freimer EH: Autoimmune mechanisms in the pathogenesis of Rheumatic fever. Rev Infect Dis 6: 832-9, 1984.

2. Cheadle WR: Harveian lectures on the various manifestations of the rheumatic state as exemplified in childhood and early life. Lancet 371: 821-7, 1889.

3. Ayoub EM: The search for host determinants of susceptibility to rheumatic fever: the missing link. Circulation 69: 197-201, 1984.

4. Paul JR: The rheumatic family. In: The epidemiology of rheumatic fever. New York, American Heart Association, chapter 14: 125-30, 1957.

5. Ayoub EM, Barret DJ, Maclaren NK, Krischer JP: Association of class II human histocompatibility leukocyte antigens with rheumatic fever. J Clin Invest 77: 2019-26, 1986.

6. Visentainer JE, Pereira FC, Dalalio MM, Tsuneto LT, Donadio PR, Moliterno RA: Association of HLA-DR7 with rheumatic fever in the Brazilian population. J Rheumatol 27: 1518-20, 2000.

7. Olmez U, Turgay M, Ozenirler S, et al: Association of HLA class I and class II antigens with rheumatic fever in a turkish population. Scand J Rheumatol 22:49-52, 1992.

8. Puttick AH, Briggs DC, Welsh KI, et al: Genes associated with rheumatoid arthritis and mild inflammatory arthritis. I Major Histocompatibility complex class I, II and III allotypes. Ann Rheum Dis 49: 219-24, 1990

9. Schroder R, Zander H, Andreas A, Mauff G: Multiple sclerosis: immunogenetic analysis of sibpair double case families II. Studies on the association of the multiple sclerosis with $\mathrm{C} 2, \mathrm{C} 4, \mathrm{BF}, \mathrm{C} 3, \mathrm{C} 6$ and Gm polymorphism. Immunobiology 164: 160- 70, 1983.
C4A*6 foi observado entre pacientes com hanseníase virchiowiana na Tailândia ${ }^{(32)}$. Há evidências de que $\mathrm{C}_{4} \mathrm{~A}^{*} 6$ não se liga eficientemente à $\mathrm{C} 3 \mathrm{~b}$ para formar a C5 convertase da via clássica ${ }^{(33)}$, impedindo a ativação desta via.

Observou-se que complexos imunes circulantes estão presentes na maioria dos pacientes com FR aguda ${ }^{(34)}$, podendo-se sugerir que fragmentos de $\mathrm{C} 4$ de alótipos A6 estariam envolvidos na ineficiente solubilização e opsonização de complexos imunes estreptocócicos, sendo um fator a mais de predisposição para as seqüelas cardíacas imunopatológicas da DCR. Enquanto a participação de antígenos das classes I e II do MHC na interação celular da resposta imunológica na FR é um conceito já estabelecido, alótipos de complemento poderiam exercer uma função regulatória em diferentes estágios da doença, desde o reconhecimento dos antígenos estreptocócicos até a efetiva eliminação pelo sistema efetor humoral específico. Considerando-se que neste estudo foram incluídos somente pacientes com DCR, outros estudos envolvendo pacientes com FR sem cardite, com o objetivo de esclarecer se C4A6 é um marcador para a forma cardíaca ou para a doença em si.

10. Messias IJT, Santamaria J, Brenden M, Reis A, Mauff G: Association of $\mathrm{C} 4 \mathrm{~B}$ deficiency $\left(\mathrm{C}^{4} \mathrm{~B}^{*} \mathrm{Q} 0\right)$ with erythema nodosum in leprosy. Clin Exp Immunol 92: 284-7, 1993.

11. Fielder AHL, Walport MJ, Batchelor JR, et al: Family study of the major histocompatibility complex in patients with systemic lupus erithematosus: importance of null alleles of C4A and C4B in determining disease susceptibility. Br Med J 286: 425-8, 1983.

12. Messias-Reason IJ, Urbanetz L, Pereira da Cunha C: Complement C3F and BF $\mathrm{S}$ allotypes are risk factors for Chagas Disease cardiomyopathy. Tissue Antigens 62:1-5, 2003.

13. Colten HR, Garnier G: Regulation of Complement protein Gene Expression. In: The human complement system in health and disease, eds. Volanakis JE, Frank MM, New York 217-240, 1998.

14. Geserick G, Abbal M, MauffG, Siemens I: Factor B (BF) nomenclature statement. Complement Inflamm 7: 255-260, 1990.

15. Alper CA: Inherited structural polymorphism in human C2: evidence for genetic linkage between C2 and BF. J Exp Med 144: 1111-4, 1976

16 Uring-Lambert B, Gas S, Goetz J, et al: Detection of the genetic polymorphism of human $\mathrm{C} 2$ (native proteinand $\mathrm{C} 2 \mathrm{a}$ fragment) by immunoblotting after polyacrylamide gel isoeletric focusing. Complement 2: 185-92, 1985.

17. Mauff G, Alper CA, Dawkins R, et al: C4 nomenclature statement (1990). Complement Inflamm 7: 261-8, 1990.

18. Doxiadis G, Grosse-Wilde H: Allotyping by prolonged gel electrophoresis and immunoblotting using monoclonal and polyclonal antibodies. Complement Inflamm 7: 269-76, 1990.

19. Mauff G: Application of the MHC-class III complement markers to populations genetics. In Ohayon E, Cambon-Thomsem A (Ed). Human Population Genetics. Paris, Les Editions INSERM, 143-66, 1986. 
20. Clegert-Darpoux F, Babron MC, Prum B, Lathrop GM, Deschamps I, Hors J: A new method to test genetic models in HLA associated diseases: The MASC method. Ann Hum Gen 52: 247-58, 1988.

21. Patarroyo ME, Winshester RJ, Vejerano A, et al: Association of a B-cell alloantigen with susceptibility to rheumatic fever. Nature 278: 173-4, 1979.

22. Khanna AK, Buskirk DR, Williams RC Jr, et al: Presence of a non-HLA B cell antigen in Rheumatic Fever Patients and their families as defined by a monoclonal antibody. J Clin Invest 83: 1710-6, 1989.

23. Anastasiou-Nana M, Anderson JL, Carlquist JF, Nanas JN: HLADR typing and lymphocyte subset evaluation in rheumatic heart disease: A search for immune response factors. Am Heart J 112: 992-7, 1986.

24. Harel L, Zeharia A, Kodman Y, Straussberg R, Zabriskie JB, Amir $\mathrm{J}$ : Presence of the $\mathrm{d} 8 / 17 \mathrm{~B}$-cell marker in children with rheumatic fever in Israel. Clin Genet 61: 293-8, 2002.

25. Caughey DE, Douglas R, Wilson W, Hassal IB: HLA antigens in Europeans and Maoris with rheumatic fever and rheumatic heart disease. J Rheumatol 2: 319-22, 1975.

26. Gorodezky C, Ulloa-LS, Escobar-Gutierrez A: Hla antigens and rheumatic heart disease in Mexico (abstract). J Rheumatol 4 (Suppl 3): 112, 1977.
27. Leirisalo M, Laitinen O, Tiilikainen A: HLA phenotypes in patients with rheumatic fever, rheumatic heart disease and Yersinia arthritis. J Rheumatol 4 (Suppl 3): 78-83, 1977.

28. Messias IJT, Reis A, Almeida PT, Mauff G: Genetic variability of the MHC Class III Complement proteins C2, BF, C4A and C4B in Southern Brazil. Exp Clin Immunogenet 11: 192-6, 1994.

29. Saeland E, VidarssonG, Leusen JH, et al: Central role of complement in passive protection by human IgG1 and IgG2 anti-pneumococcal antibodies in mice. J Immunol 170: 6158-64, 2003.

30. Jarva H, Jokiranta TS, Wurzner R, Meri S: Complement resistance mechanisms of streptococci. Mol Immunol 40 (2-4): 95-107, 2003.

31. Alper CA, Rosen FS: Inherited deficiencies of complement proteins in man. Springer Semin Immunopathol 7: 251-61, 1984.

32. Greiner J, Weber FJ, Mauff G, Bauer MP: Genetic popymorphism of properdin factor $\mathrm{B}(\mathrm{Bf})$, the second component $(\mathrm{C} 2)$, and the fourth component $(\mathrm{C} 4)$ of component in leprosy patients and healthy controls from Thailand. Immunobiology 158:134-8, 1980.

33. Dodds AW, Law SK, Porter RR: The origin of the very variable haemolytic activities of the common human complement component C4 allotypes, including C4A6. EMBO J 4:2239-44, 1985.

34. Yoshinoya S, Pope RM: Detection of immune-complexes in acute rheumatic fever and their relationship to HLA B5. J Clin Invest 65:136-45, 1980. 